\title{
Wireless Sensor Data Collecting to MobileCloud Device
}

\author{
Shashank Shukla* \\ Anil Kumar Gupta** \\ Research Scholar \\ Department of $\mathrm{IT}^{\star}, \mathrm{CSE}^{* *}$ \\ GCET Greater Noida UP
}

(INDIA)

\author{
Santosh Kumar \\ Upadhyay \\ Asstt. Prof. Department of CSE \\ GCET Greater Noida UP \\ (INDIA)
}

\author{
Ashu Gupta \\ Android Developer \\ Matter of Tech. Pvt. Ltd \\ Gurgaon \\ HARYANA (INDIA)
}

\begin{abstract}
Mobile devices are becoming a part of life in this new era. These days' smartphone having a lots of Applications which make the work very easy and smooth. There is huge amount of mobile applications for different - different categories. Smartphone application also integrate with other hardware and collects data or information from hardware.Wireless Sensor networks provides a lot of contribution in the daily life of users. One of the common applications of Wireless Sensor Network is to indulge in security of household things such as bikes, cars and home security. Cloud computing is one of the popular technology in the category, it will be going to capture all industries. Cloud vendors will go to look after work of the industrieswith growth in Wireless Sensor application's.It alsosupports in recognition of natural calamities like flood detection, fire detection and various other applications, but it fails to deliver dynamism in detection of sensing data. With dynamism provided in cloud computing and wireless sensor network integrates with cloud. As smart phone application with android platform is in rebellionthese days, combination of all this technology makes the availability of data dynamically at mobile phone. In this paper we going to implement integration of all this technology to get right amount of data instantly as smart phone application.
\end{abstract}

\section{Keywords}

Cloud computing, Wireless Sensor, Android.

\section{INTRODUCTION}

Currentmethods for gathering data require a great deal of work that requires large amount laborious and physical work. Collecting information requires dealing with hard complexity [3] of network in wireless sensor network. Wireless sensor network is functional field where a human cannot reach like in deepjungle to sense fire, flood sensitive where humanbeing cannot present for long to regulate the level of water. So, distribution of sensors in such places makes it familiar to get data from such remote seats which [1] are unreachable from human. Wireless sensor network also give application in GPS recognition of user position according to longitudinal and latitude data provided by the satellites.

The Wireless Sensor Network is erected of nodes from a few to thousands or hundreds, where each node is connected to one or numerous sensors. Individuallyall such sensor network node has normally several parts, a microcontroller, relating to an external investigation or a radio transceiver [2] with an internal antenna, an electronic circuit for interfacing with the sensors and an energy source and a battery. The price of sensor nodes is similarly variable, oscillatinga rare to hundreds of dollars, contingent on the complexity of the individual sensor nodes. Size and cost constraints on sensor nodes result in consistent on resources such as recollection, energy, computational speed and communications bandwidth.
The topology of the WSNs can vary from a artless star network to an advanced multi-hop wireless mesh network. The proliferationmethod between the hops of the network can be routing. Sensor Network prototype is shown below in figure 1.

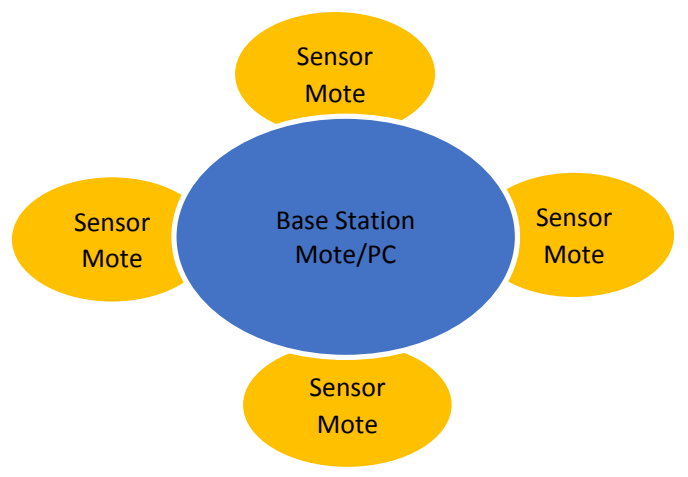

Figure-1: Sensor Network

There are applications that can be advanced the usage of sensor networks and a node. Sensing applications smooth flexible management of heating, lighting, and cooling systems from anywhere in the home. [5] Sensing applications also allow one to arrange and run numerous systems from a single remote control. Sensing applications, simplify the reaction of automatic announcement upon detection of unusual events.

\section{CLOUD COMPUTING}

Cloud Computing is a trendy model in Information Technology today. It provides lots of services instantly. As it becoming popular, it is also becoming more challenging and developing area of computing. [9]Many cloud providers are now active in the market, providing a rich offering several kind of services to the user. The cloud technology has become mainstream in enterprise data centers, where private, public and hybrid cloud architectures. [10] Cloud computing is the delivery of computing and storing bulk as to a communal of end-recipients. [3]Cloud computing permit you to use data or application from other than your workstation or internet connected device most often it will be a withdrawn data center. Splendor of cloud is other firms can host the application which means that they can handle the cost of servers, they manage the software updates. [4] 


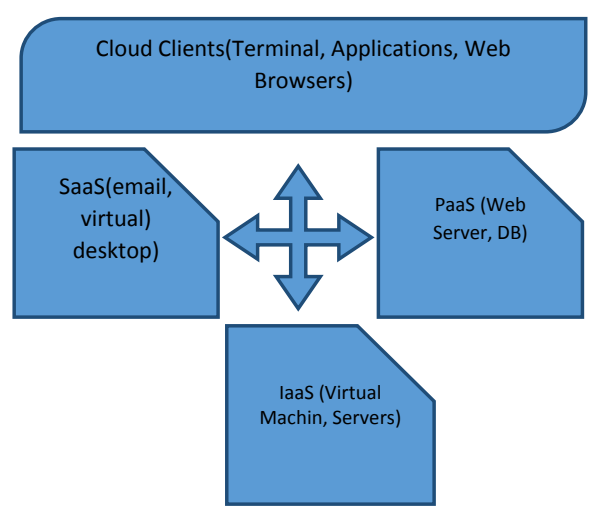

Figure-2 Cloud Service Model

\section{ANDROID}

Android is a mobile OSwhich is foundedbyanimproved version of Linux. It was originallydemonstrated by a startup of the same, Android, Inc. Itis a part of its strategy to enter the mobile space, in 2005. Google purchased Android and acquiredits development work. [7] Google desired Android to be vulnerable and unrestricted; hence, most of the Android code was released under the open-source Apache License, which means that anyone who wants to use Android can do so by copying the full Android code. Moreover, vendors can add their own named extensions to Android and customize [8] Android to differentiate their products from others. Android's source code is released by Google under open source licenses, although most Android devices eventually ship with a mixture of open source and proprietary software, including proprietary software required for accessing Google services.

\section{INTEGRATION OF WIRELESS SENSOR NETWORK TO CLOUD COMPUTING AND ANDROID}

\subsection{Deployment Of The Sensor}

Wireless sensor is completed by addition of motes through the network for collecting information from the outside-world. Sensors acquire the surroundings readings data where they deployed. [1] When the readings data is taken, it is transferred to the base station. The Rssi Demo and BlinkToRadio application gives the illustration readings data for the communication among the sensor motes and motes to PC. Mote connected is act as the base station for the motes installed in the sensor networks. All the readings gathered is transfer to the base station mote and from base station it will drives to PC. Computer system related with the base mote gets the data[2] data and saves it to the hard disk. If large amount of data is coming from the sensors, then it will hand over it to the cloud environment.

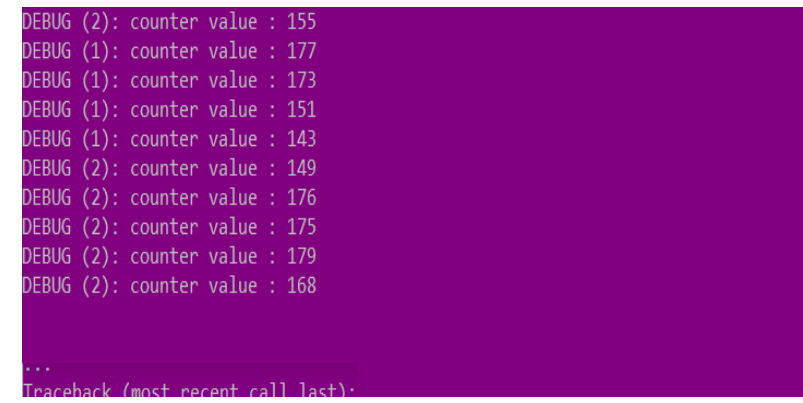

Figure-3 Showing the readings from the mote to mote communication
Illustrated data from the mote to pc communication is shown by the following output.[15]

00 FF FF 000100040600010001

00 FF FF 000100040600010002

00 FF FF 000100040600010003

00 FF FF 000100040600010004

00 FF FF 000100040600010005

00 FF FF 000100040600010006

00 FF FF 000100040600010007

00 FF FF 000100040600010008

00 FF FF 000100040600010009

00 FF FF $00010004060001000 \mathrm{~A}$

Cloud-computing, provides the storage area for the collected data from coming sensor readings. The information from sensor networks is stored in a cloud platform and made accessible for the smart phone application. [16]

\subsection{Integration Of Sensor Over Cloud Computing}

Cloud computing delivers an easy way of packing the information from one platform to cloud. In cloud computing resources are provided by the cloud vendors like providing processing memory for the work of end users. Once you are in cloud you need not have to concern about your work. Cloud computing delivers three basic attributes PaaS (Platform as a Service), SaaS (Software as a Service) and IaaS (Infrastructure as a Service). Along with this three attributes public cloud, private cloud and also Hybrid cloud are there for providing services to users. Public cloud is for providing users to connect their platform with users at distant places like Google drive, Google docs are the example of the public cloud. [18] Private clouds are being setup within some organization. Suppose you are running some industry than you have to provide some memory space to each of your employee, accessing space for software.All these things are done by setting up the private cloud environment. Hybrid clouds are the combination of both public and private cloud. [11]

\subsection{Mobile Application}

Android/IOS application in present day is a big revolution in mobile computing. Mobile computing contains development of operating systems and also the application context for the mobile handheld systems. [19] Google android is one of the rebellions in this field. Application development in android platform is cool for those who are in touch with the Android Studio. Android Studio provides the environment for the development of the android based application.[20]

\subsection{Architecture Of The System}

The architecture contains of shifting of data from sensor network to cloud platform and transfer the readings from cloud platform to android application. The idea proposed in this paper designs the application for android mobile phone for monitoring the sensors at distant places.[21] Places like fire sensitive forests where the possibility of fire is high and it can be very intenselynoticeable by the mobile application.

\section{RELATED WORK}

The application of mobile platform is for pervasive healthcare information management has already been acknowledged and 
well established ([2], [6]). Authors in [5] contemporaneous the assistances of using virtual health records for mobile care of ageing citizens. The keydetermination of the work is to provide unified and consistent communication flow between home health care and main care wage-earners using devices like PDAs and Tablet PCs. Smart cards andWeb interfaces have been used in [3] for storing patients records electronically. The MADIP system [4] is a scattered information platform allowing wide-area information exchange based on mobile agents. In [7] authors present a moveable platform for exchanging their images and records over wireless networks using progressive compression schemes. The majority of theabove-mentioned works is based on proprietary architectures and communication schemes and requires the deployment of explicit software components. Also, the works focus mostly on transporting data to healthcare applications and do not discourseproblems of data management and interoperability issues introduced by the varied data resources found in current healthcare systems. The usage of Cloud Computing provides data management and access functionality overcoming the above-mentioned issues asdiscussed in preceding sections. The concept of using Cloud Computing in the setting of healthcare information management is relatively new but is careful to have great potential [8]. To our best knowledge there is no other work in the works utilizing Cloud Computing for providing pervasive healthcare information management services on mobile devices.

\section{APPLICATION FRAMEWORK}

In this segmentdiscourses the key features of the application which associate all the three technologies organized. Initially, wireless sensor network acquires the information data from the field where sensor motes get deployed. Supposing sensors were deployed in the fire sensitive places, so by collecting the infection readings from the sensors we can get the realsituation of the place. For this we are using Tiny-OS for programming sensor motes into nesC language and install amassed program into motes. Cloud storing will deliver a large amount ofmemory space for storing and retrieving the data. There are sufficiently of sellers providing storing space for your documents into their space. Onedrive, DropBox,Google drive are few of the sellers providing limited free space over the cloud environment. In our application we are using Drop Box for storing sensor readings data and also for saving the information to dropbox over the cloud. [22]

\section{PROPOSED SYSTEM AND IMPLEMENTATION}

The completedesign of the application associates the wireless sensor network with the cloud and android for reclaiming values from the cloud. The architecture is shown in the figure 4

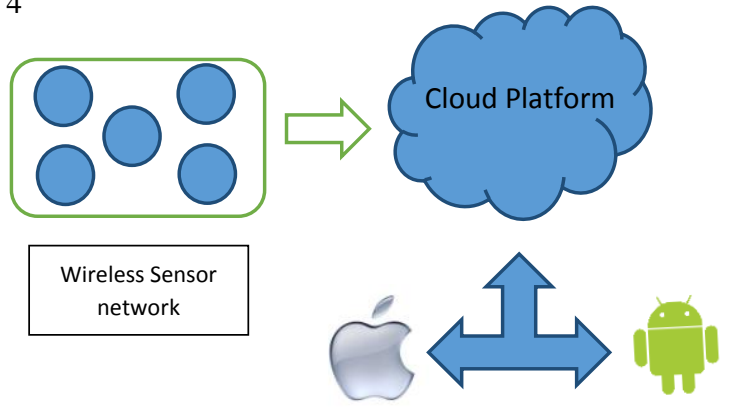

Figure- 4 Simplified overall architecture
Overhead design consists wireless sensor network with sensor motes. Motes are installed with programs for responsibility the work in the place specified. nesC coding is compiled and fixed in the motes. For this we have to install the TinyOS platform for the sensors in Ubuntu. For simulation, we are taking basic applications to show the outputs. Sensors motes are get installed by typing the command make telosb 1 where 1 mote id and telosb is the name of the sensor mote. For simulation, we are consuming TOSSIM simulator on TinyOs. For TOSSIM we have to import tossim library in our program-model and also we have to add a command to show the output to the screen. Once we got the output, we can save it by getting the output reading inside a text file. After getting the output in the text file store this text file into the cloud environment. By installing DropBox we can store the readings over the cloud. For this we to make an account into DropBoxthrough drobox official website.[14][15] Drop Box will provide to user $2 \mathrm{~GB}$ of free space. For simulation drive we are using such a small size of memory. For android, install Android Studio for the development. After installation connect it with the android latest Android SDK version. After development run the application as android application. The Application retrieves the data coming from the sensor by accessing the cloud; also it will give the alert message to user when the data reading goes beyond the thresholdvalue.

\section{CONCLUSION}

It is a minor application achieves to monitor places which are very sensitive to some kind of natural disasters and also it can be effortlesslyadaptable for using in other application like household, cars, bike etc.

There are lots of application which can be integrate to the wireless sensors and mobile platform through cloudcomputing and make the human work very easy and fatly.

\section{REFERENCES}

[1] Kwang-il Hwang, JeongsikIn, NhoKyung Park, DooseopEom -A Design and Implementation of Wireless Sensor Gateway for Efficient Querying and Managing through World Wide Webll.

[2] Sabine Koch, Maria Hägglund, Isabella Scandurra, Dennis Moström, towards a virtual health record for mobile home care of elderly citizens, presented in MEDINFO 2004, Amsterdam, 2004.

[3] Alvin T.S. Chan, -WWW smart card: towards a mobile health caremanagement systemll, International Journal of Medical Informaticsvol. 57, 2000, pp. 127137.Maglogiannis I., Doukas C., Kormentzas G., Pliakas T., "Wavelet-Based Compression With ROI Coding Support for Mobile Access toDICOM Images Over Heterogeneous Radio Networks", IEEETransactions on Information Technology in Biomedicine, vol.13, no.4,pp.458-466, July 2009.

[4] Khawar Hameed, - The application of mobile computing andtechnology to health care servicesl, elematics and Informatics, vol.20, 2003, pp. 99-106.

[5] Eneida A. Mendonça, Elizabeth S. Chen, Peter D. Stetson, LawrenceK. McKnight, Jianbo Lei, James J. imino, -Approach to mobileinformation and communication for health carell, InternationalJournal of Medical Informatics, vol 73, 2004, pp. 631-638.

[6] Chuan Jun Su, -Mobile multi-agent based, distributed informationplatform (MADIP) for wide-area e-health 
monitoring\|, Computers inIndustry, vol. 59, 2008, pp. $55-68$.

[7] Quality-of-service in cloud computing: modeling techniques and their applications Danilo Ardagna, Giuliano Casale, Michele Ciavotta, JuaJuann F PérezandWeikun Wang in 2014.

[8] OferShimrat, -Cloud Computing and Healthcarell, April 2009 "San Diego Physician", pp. 26-29.

[9] Shashank Shukla, Anil Kumar Gupt, Sandeep Saxena, Santosh Kumar Upadhyay "An Evolutionary Study of Multi-Objective Workflow Scheduling in Cloud Computing" in International Journal of Computer Applications (0975 - 8887)Volume 133 - No.14, January 2016.

[10] Anil Kumar Gupta, Shashank Shukla, Sandeep Saxena, Sanjay Khakhil "A Journey Towards Workflow Scheduling of Cloud Computing" in International Journal of Computer Applications (0975 - 8887) Volume 123 No.4, August 2015.

[11] C. Intanagonwiwat, R. Govindan, and D. Estrin, "Directed diffusion: a scalable and robust communication paradigm for sensor networks," Proceedings of ACM MobiCom '00, Boston, MA, 2000, pp. 56-67.

[12] D. Braginsky and D. Estrin, \Rumor Routing Algorithm for Sensor Networks," in the Proceedings of the First Workshop on Sensor Networks and Applications (WSNA), Atlanta, GA, October 2002.

[13] F. Ye, A. Chen, S. Liu, L. Zhang, \A scalable solution to minimum cost forwarding in large sensor networks",
Proceedings of the tenth International Conference on Computer Communications and Networks (ICCCN), pp. 304-309, 2001.

[14] C. Schurgers and M.B. Srivastava, IEnergy efficient routing in wireless sensor networks", in the MILCOM Proceedings on Communications for Network-Centric Operations: Creating the Information Force, McLean, VA, 2001.

[15] Amirante, Castaldi, Miniero and Romano, "Meetecho Mobile: Accessing an IETF-compliant conferencing framework from cellular devices", Communications Magazine, (2011), pp. 36 - 43.

[16] C. Wang, W. Duan, J. Ma and C. Wang, "The research of Android System architecture and application programming”, ICCSNT, (2011), pp. 785 - 790

[17] Leonard Richardson, Sam Ruby, and David HeinemeierHansson,Restful Web Services, Paperback, O'Reilly Media, May 2007, ISBN:0596529260.

[18] The Digital Imaging and Communications in Medicine (DICOM)

[19] US Secure Hash Algorithm 1 (SHA1),http://www.faqs.org/rfcs/rfc3174.html

[20] Eric Rescorla, SSL and TLS: Designing and Building Secure Systems(Paperback), Addison-Wesley Professional (October 27, 2000), ISBN:0201615983.

[21] The SQLite Database Engine, http://www.sqlite.org/

[22] Creating HIPAA-Compliant Medical Data Applications with Amazon. 